\title{
Scaling and universality in turbulent convection
}

\author{
Antonio Celani ${ }^{1}$, Takeshi Matsumoto ${ }^{2,3}$, Andrea Mazzino ${ }^{4,5}$, and Massimo Vergassola ${ }^{2}$ \\ 1 CNRS, INLN, 1361 Route des Lucioles, 06560 Valbonne, France. \\ ${ }^{2}$ CNRS, Observatoire de la Côte d'Azur, B.P. 4229, 06304 Nice Cedex 4, France. \\ ${ }^{3}$ Dept. of Physics, Kyoto University, Kyoto 606-8502, Japan. \\ ${ }^{4}$ CNR-ISIAtA, Polo Scientifico dell Università, I-73100, Lecce, Italy. \\ ${ }^{5}$ INFM-Dipartimento di Fisica, Università di Genova, Via Dodecaneso, 33, I-16146 Genova, Italy.
}

(Dated: November 13, 2018)

\begin{abstract}
Anomalous correlation functions of the temperature field in two-dimensional turbulent convection are shown to be universal with respect to the choice of external sources. Moreover, they are equal to the anomalous correlations of the concentration field of a passive tracer advected by the convective flow itself. The statistics of velocity differences is found to be universal, self-similar and close to Gaussian. These results point to the conclusion that temperature intermittency in two-dimensional turbulent convection may be traced back to the existence of statistically preserved structures, as it is in passive scalar turbulence.
\end{abstract}

PACS numbers: 47.27.-i

Heat and momentum transport in slightly heated flows are governed by the Boussinesq equations [1]

$$
\begin{gathered}
\partial_{t} T+\boldsymbol{v} \cdot \boldsymbol{\nabla} T=\kappa \Delta T+f_{T} \\
\partial_{t} \boldsymbol{v}+\boldsymbol{v} \cdot \boldsymbol{\nabla v}=-\boldsymbol{\nabla} p-\beta T \boldsymbol{g}+\nu \Delta \boldsymbol{v},
\end{gathered}
$$

where $T$ is the field of the temperature fluctuations, $v$ is the velocity field, $\boldsymbol{g}$ is the gravitational acceleration, $\beta$ is the thermal expansion coefficient and $\kappa, \nu$ are the molecular diffusivity and viscosity. The system is kept in a statistically stationary state by the external source of fluctuations $f_{T}$. We will focus on the statistical properties of temperature excursions at scales larger than the Bolgiano scale $l_{B}$ - where buoyancy forces balance the inertial ones in the velocity dynamics - yet smaller than the forcing correlation length, $L$. In that range, temperature fluctuations cascade toward the small scales where they are eventually dissipated by thermal diffusivity. Dimensional arguments based on this phenomenological picture would lead to the Bolgiano-Obukhov scaling, in the range $l_{B} \ll r \ll L: S_{n}^{T}(r)=\left\langle[T(\boldsymbol{r}, t)-T(\mathbf{0}, t)]^{n}\right\rangle \sim r^{n / 5}$, and $S_{n}^{v}(r)=\left\langle[(\boldsymbol{v}(\boldsymbol{r}, t)-\boldsymbol{v}(\mathbf{0}, t)) \cdot \hat{\boldsymbol{r}}]^{n}\right\rangle \sim r^{3 n / 5}$ (see, e.g., Ref. [2] and references therein). Actually, due to the presence of structures of warm-rising or cold-descending fluid - the thermal plumes (see Fig. 1) - the statistics of temperature increments exhibits a nontrivial scale-dependence. Indeed, as shown in Figure 2, moments of temperature increments display a scaling behavior $S_{n}^{T}(r) \sim r^{\zeta_{n}^{T}}$ characterized by exponents deviating from the dimensional expectations. (Conversely, moments of velocity increments, $S_{n}^{v}(r) \sim r^{\zeta_{n}^{v}}$, do not show measurable deviations from dimensional scaling, i.e. $\zeta_{n}^{v}=3 n / 5$.) This anomalous scaling is a feature shared by a large class of turbulent systems: understanding the origin of this phenomenon from first principles is a major challenge of turbulence. With a significant exception. Indeed, for passive turbulent transport this problem has been recently solved. Let us briefly recall the main points, referring to Ref. 3 for a comprehensive review. We consider an idealized ex- periment of turbulent dispersion of a passive tracer, e.g. dye, in a convective flow. The equation that governs the dynamics of the concentration of tracer is

$$
\partial_{t} C+\boldsymbol{v} \cdot \nabla C=\kappa \Delta C+f_{C},
$$

while the velocity field $\boldsymbol{v}$ evolves according to Eq. (1). Although seemingly similar, the dynamics of temperature and concentration fields are radically different: temperature is an active scalar, since it affects velocity via

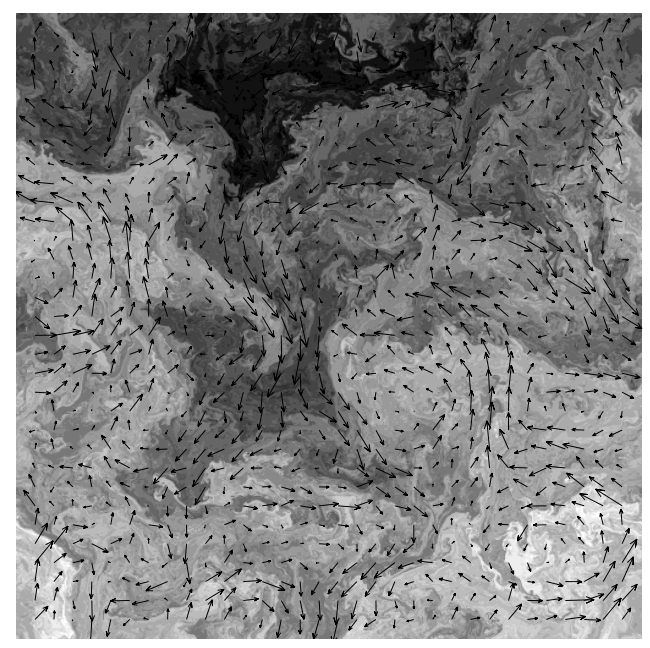

FIG. 1: Snapshot of the temperature and velocity fields. Dark areas identify cold regions. The Boussinesq equations (1) are solved in a two-dimensional doubly periodic domain, with $1024^{2}$ collocation points. The Bolgiano scale $l_{B}$ is comparable to the smallest resolved length-scale. Since in two dimensions there is a net energy flux toward the large scales, a statistically steady state requires the momentum equation in (1) be supplemented by a friction term $-\alpha \boldsymbol{v}$ that drags energy from the gravest modes. As customary, diffusive and viscous terms are replaced by hyperdiffusive $\left(-\kappa \Delta^{2}\right)$ and hyperviscous $\left(-\nu \Delta^{4}\right)$ ones, to confine dissipative effects to the smallest scales. 
the buoyancy forces, whereas concentration is a passive scalar. The concentration fluctuations show an anomalous scaling behavior $S_{n}^{C}(r)=\left\langle[C(\boldsymbol{r}, t)-C(\mathbf{0}, t)]^{n}\right\rangle \sim r^{\zeta_{n}^{C}}$ as well: the exponents $\zeta_{n}^{C}$ differ from the dimensional expectation. Since $S_{n}^{C}(r)$ is a linear combination of various $n$-point correlation function of the concentration field $\left\langle C\left(\boldsymbol{x}_{1}, t\right) \ldots C\left(\boldsymbol{x}_{n}, t\right)\right\rangle$, the latter has to contain a contribution, denoted as $Z_{n}^{C}\left(\boldsymbol{x}_{1}, \ldots, \boldsymbol{x}_{n}\right)$, that carries the anomalous scale dependence. In mathematical terms, $Z_{n}^{C}\left(\lambda \boldsymbol{x}_{1}, \ldots, \lambda \boldsymbol{x}_{n}\right)=\lambda^{\zeta_{n}^{C}} Z_{n}^{C}\left(\boldsymbol{x}_{1}, \ldots, \boldsymbol{x}_{n}\right)$. The main point is that the function $Z_{n}^{C}$ is characterized by a special dynamical property that distinguishes it from a generic scaling function. Let us remind the passive scalar equation (2) can be written in the equivalent form $\frac{d}{d t} C=f_{C}$, where $\frac{d}{d t}$ stands for the total derivative along the particle trajectories defined by the stochastic differential equations $d \boldsymbol{X}=\boldsymbol{v}(\boldsymbol{X}, t) d t+\sqrt{2 \kappa} d \boldsymbol{W}(t)$, where $\boldsymbol{W}(t)$ is brownian motion. The remarkable result is that $\frac{d}{d t}\left\langle Z_{n}^{C}\right\rangle_{X}=0$, where the total derivative is performed following $n$ particles advected by the flow, and the average is taken over the ensemble of all trajectories. In plain words, $Z_{n}^{C}$ is statistically preserved by the flow $[5,6,7]$. In the specific context of a Gaussian, $\delta$-correlated velocity field - the Kraichnan model - this is equivalent to say that the functions $Z_{n}^{C}$ are zero-modes of the FokkerPlanck operator for $n$-particle diffusion $[8,9,10]$. An important consequence of statistically preserved structures is that the passive scalar scaling exponents are universal with respect to the choice of the injection $f_{C}$, since the latter does not enter the definition of $Z_{n}^{C}$.

We now turn our attention back to the temperature field. What we have learnt in the passive scalar case suggests

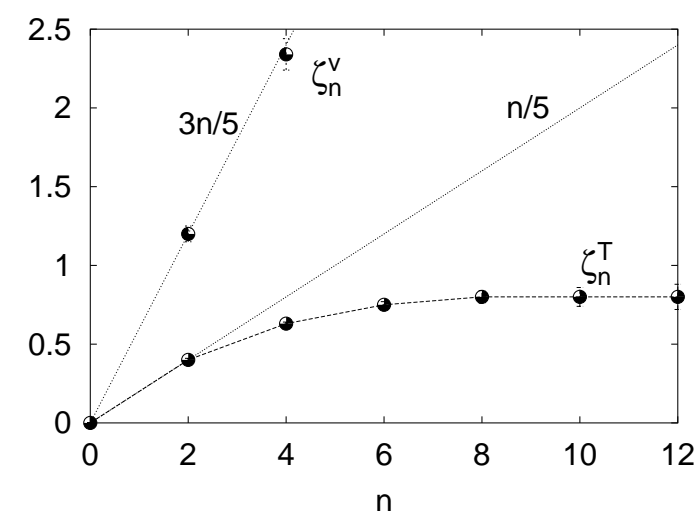

FIG. 2: Scaling exponents of temperature, $\zeta_{n}^{T}$, and velocity, $\zeta_{n}^{v}$. The straight lines are the dimensional predictions, $n / 5$ for temperature, $3 n / 5$ for velocity. Notice that at orders larger than $n=8$ the temperature exponents saturate to a constant value $\zeta_{\infty}^{T} \simeq 0.8[4]$. The errorbars are estimated by the rms fluctuations of the logarithmic slope. To ensure the statistical convergence of high-order moments we collected three hundreds of snapshots of the fields, spaced by half of the largeeddy turnover time $L /\left\langle\boldsymbol{v}^{2}\right\rangle^{1 / 2}$. to investigate the effect of the external forcing $f_{T}$ on the scaling exponents. In Fig. 3 we show that the scaling exponents of temperature fluctuations are the same for two different choices of injection terms $f_{T}$. Therefore, we conclude that the exponents $\zeta_{n}^{T}$ are universal properties of two-dimensional Boussinesq convection. The univer-

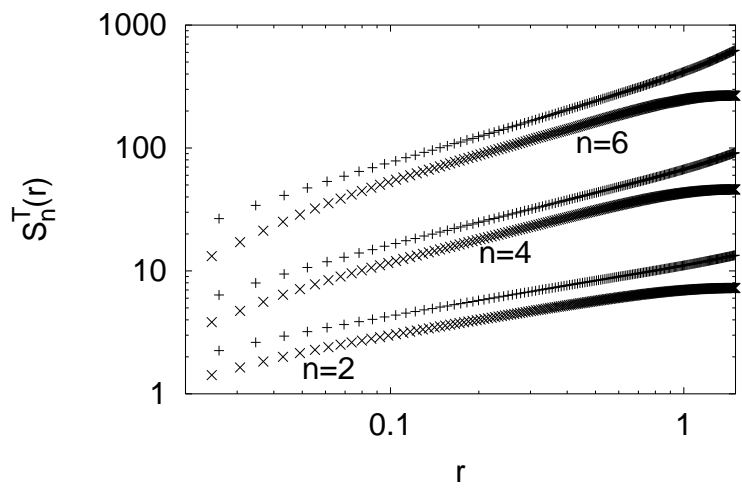

FIG. 3: The moments of temperature differences, $S_{n}^{T}(r)$, for $n=2,4,6$, as a function of the separation $r$. Note the parallelism between curves of the same order $n$, within the scaling range. The two sets of curves are generated by two different kinds of injection mechanisms. In the first case $(\times), f_{T}$ is a random Gaussian forcing, with correlation $\left\langle f_{T}(\boldsymbol{r}, t) f_{T}\left(\boldsymbol{r}^{\prime}, t^{\prime}\right)\right\rangle=F_{T}\left(\left|\boldsymbol{r}-\boldsymbol{r}^{\prime}\right|\right) \delta\left(t-t^{\prime}\right)$, where $F_{T}$ decays with the characteristic scale $L$ (approximately one-fourth of the box-size); in the second case $(+)$, the system is driven by the term $f_{T}=\gamma \boldsymbol{g} \cdot \boldsymbol{u}$, that mimics the effect of a mean temperature gradient on the transport of temperature fluctuations. We consider only the isotropic contribution to the statistics, by averaging over all directions of the separation $\boldsymbol{r}$. For orders equal or larger than $n=8$ all exponents collapse - within errorbars - on the saturation value $\zeta_{\infty}^{T} \simeq 0.8$. The curves have been multiplied by appropriate numerical factors for viewing purposes. The equality of the scaling exponents $\zeta_{n}^{T}$ for the two types of forcing has been checked by computing the logarithmic slope $d \ln S_{n}^{T}(r) / \ln r$ (not shown).

sality of scaling exponents suggests the possibility that a mechanism similar to that at work in passive scalar turbulence might be present in turbulent convection as well. To further pursue this line of thought, we notice that in the case of passive scalars, it is the whole function $Z_{n}^{C}$ to be universal with respect to forcing, not only its scaling exponent. It is thus of interest to look at the anomalous part of the temperature correlation function $\left\langle T\left(\boldsymbol{x}_{1}, t\right) \ldots T\left(\boldsymbol{x}_{n}, t\right)\right\rangle$, to check whether it is universal. This measurement is unfortunately quite difficult for two reasons. First, the correlation function depends on $2 n$ independent coordinates; even if we exploit the statistical symmetries of this function - translational and scaling invariance - and we limit ourselves to the isotropic contribution - by averaging over all configurations differing only by a rigid rotation - there will still be $2 n-4$ degrees of freedom; in the most favorable case, $n=4$ (for $n=2$ 

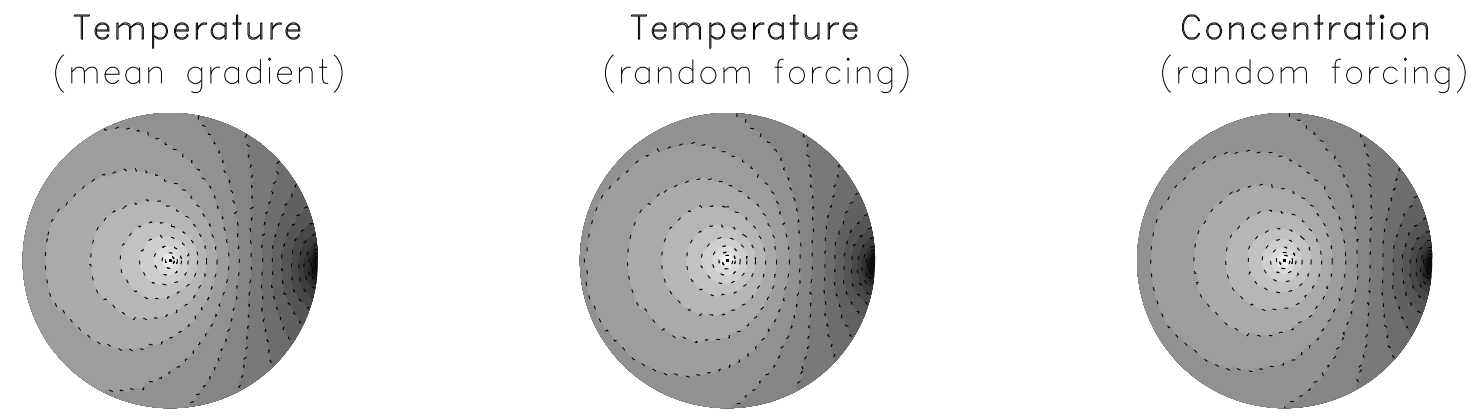

FIG. 4: The functions $\sigma_{T}(r / R, \theta)=S_{2,2}^{T}(\boldsymbol{R}, \boldsymbol{r}) / S_{4}^{T}(R)$ (left and center), and $\sigma_{C}(r / R, \theta)=S_{2,2}^{C}(\boldsymbol{R}, \boldsymbol{r}) / S_{4}^{C}(R)$ (right), in polar coordinates $0<r / R<1$ and $0<\theta<2 \pi$, where $\theta=\cos ^{-1}(\hat{\boldsymbol{R}} \cdot \hat{\boldsymbol{r}})$. The color is white where the function is zero, black where it is equal to unity. The function has a minimum at the origin, $\sigma(0, \theta)=0$, and a maximum $\sigma(1,0)=1$ at $\boldsymbol{r}=\boldsymbol{R}$.

deviations from dimensional scaling are not detectable), the configuration space has four dimensions, which makes it quite untractable. Second, the anomalous part of the correlation function is hidden among several other contributions: it can be extracted only by taking proper linear combinations, as, for example, in the case of $S_{n}^{T}(r)$. To circumvent, at least partially, those problems, we focus on a particular observable, $S_{2,2}^{T}(\boldsymbol{R}, \boldsymbol{r})=\langle[T(\boldsymbol{R}, t)-$ $\left.T(\mathbf{0}, t)]^{2}[T(\boldsymbol{r}, t)-T(\mathbf{0}, t)]^{2}\right\rangle$, which is still anomalous, yet it has a nontrivial geometrical content. Since for $\boldsymbol{r}=\boldsymbol{R}$ it reduces to the usual $S_{4}(R)$ we can write its functional dependence as $S_{2,2}^{T}(\boldsymbol{R}, \boldsymbol{r})=S_{4}^{T}(R) \sigma_{T}(r / R, \theta)$ where $\theta$ is the angle between the directions of $\boldsymbol{r}$ and $\boldsymbol{R}$. Since the scaling exponent of $S_{4}^{T}(R)$ is universal, the bottom line is whether the "angular" part $\sigma_{T}(r / R, \theta)$ is universal as well. In Fig. 4 we show a plot of the function $\sigma$ for the two different injection mechanisms (left and center). The similarity between the two pictures points to the conclusion that the anomalous part of the correlation function is again universal.

This result leads us to conjecture that statistically preserved structures $Z_{n}^{T}\left(\boldsymbol{x}_{1}, \ldots, \boldsymbol{x}_{n}\right)$ might exist also for temperature: it is natural to define them by the property $\frac{d}{d t}\left\langle Z_{n}^{T}\right\rangle_{X}=0$, as in the passive scalar case. Notice however that, since temperature is an active scalar, this definition does not automatically ensure the universality of $Z_{n}^{T}$. Indeed, even if the forcing does not appear explicitly in the definition of $Z_{n}^{T}$, the statistics of the trajectories $\boldsymbol{X}(t)$ in principle depends on $f_{T}$, via the action of $T$ on $\boldsymbol{v}$. Therefore, should we accept the existence of statistically preserved structures, the universality of anomalous temperature correlations requires to postulate that the whole statistics of $\boldsymbol{v}$ be universal as well. In Fig. 5 we show that this is indeed the case.

Statistically preserved structures for temperature fluctuations entail another interesting consequence: since $Z_{n}^{T}$ is defined entirely in terms of the (universal) statistics of particle trajectories, and those are the same both for temperature and for concentration, we expect that $Z_{n}^{T}=Z_{n}^{C}$. In Fig. 6 we show that the scaling exponents of temperature and concentration are equal, as expected. As for the "angular part" of $Z_{n}^{T}$, it is quite similar to that of $Z_{n}^{C}$ (see Fig. 4, center and right). This is a further indirect evidence for the existence of statistically preserved structures for temperature statistics.

In conclusion, the global picture of scaling and universality in two-dimensional turbulent convection is as follows. Velocity statistics is strongly universal with respect to the external driving: probability density functions of velocity fluctuations are self-similar, and close to a Gaussian distribution, independently of the choice of $f_{T}$. This is most likely a consequence of the observed

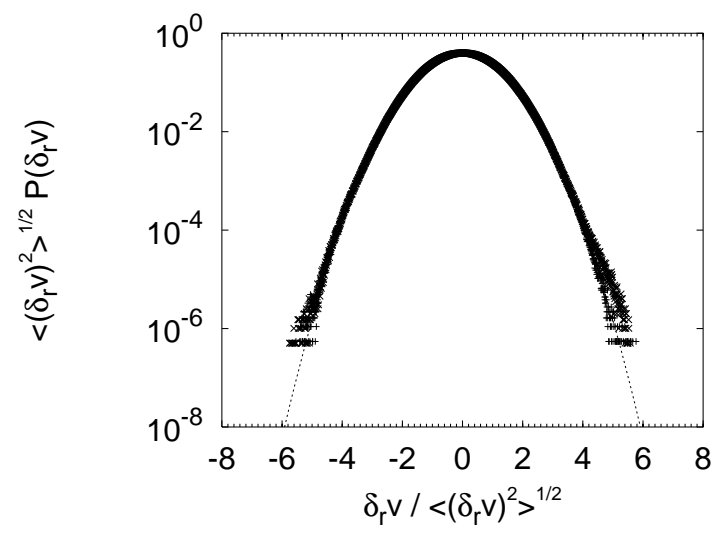

FIG. 5: Probability density function of longitudinal velocity increments $\delta_{r} v=(\boldsymbol{v}(\boldsymbol{r}, t)-\boldsymbol{v}(\boldsymbol{r}, 0)) \cdot \hat{\boldsymbol{r}}$, rescaled to their standard deviation $\left\langle\left(\delta_{r} v\right)^{2}\right\rangle^{1 / 2}$. We show two sets of data obtained by driving the system with random Gaussian forcing $(\times)$, and by $f_{T}=\gamma \boldsymbol{g} \cdot \boldsymbol{u}(+)$. Here $r=0.2$, inside the scaling range. At different $r$ the rescaled pdf's collapse onto each other, as expected for a self-similar statistics. The Gaussian density function is shown as a dotted line, for comparison. 


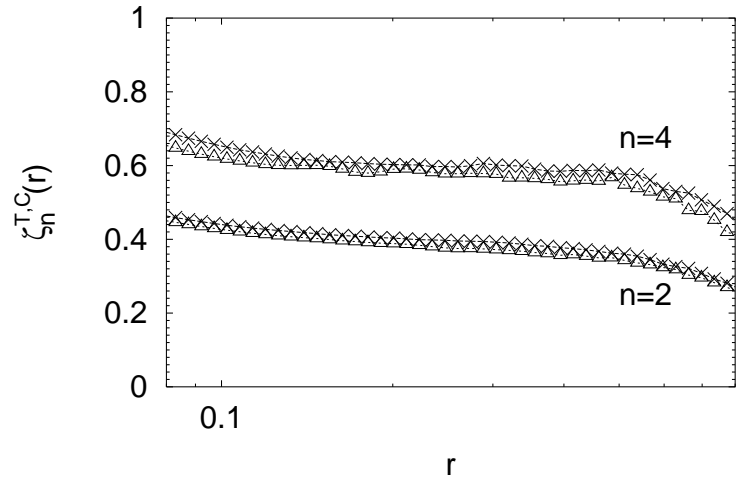

FIG. 6: Local scaling exponents of temperature $(x)$ and concentration $(\triangle)$ fluctuations, $\zeta_{n}^{T, C}(r)=d \ln S_{n}^{T, C}(r) / d \ln r$. Temperature and concentration are driven by independent Gaussian random forcings.

universal Gaussian behavior of the inverse energy cascade in two-dimensional Navier-Stokes turbulence [11, 12]. Indeed, velocity fluctuations in two-dimensional convection also arise from an inverse energy cascade which is driven now by buoyancy forces. At variance with the usual Navier-Stokes inverse cascade, the energy injection now is not restricted to small scales. Indeed, the energy input rate $\varepsilon(r)=\beta \boldsymbol{g} \cdot\langle\boldsymbol{v}(\boldsymbol{r}, t) T(\mathbf{0}, t)\rangle$ grows with the scale as $\varepsilon(r) \sim r^{4 / 5}$. This scale-dependent input induces the observed scaling $S_{n}^{v}(r) \sim(\varepsilon(r) r)^{n / 3} \sim r^{3 n / 5}$. Temperature statistics shows anomalous scaling. This stems from the existence of statistically preserved structures, whose existence explains the observed universality of anomalous temperature correlation functions and the equality between temperature and concentration anomalies.

Let us point out that the equivalence of the statistics of an active scalar, as temperature, to that of a passive scalar, as concentration, depends crucially on the universality of the whole velocity statistics found here. That could however be a nongeneric phenomenon in ac- tive scalar turbulence, and depend on the specific form of the feedback of the scalar field on the velocity. For example, in three-dimensional turbulent convection the Navier-Stokes equations are characterized by a direct and intermittent energy cascade, the whole velocity statistics might then be nonuniversal, and a new type of universality might emerge.

We acknowledge several useful discussions with R. Benzi, L. Biferale, S. Toh. This work was supported by EU under the contracts HPRN-CT-2000-00162 and FMRXCT-98-0175. A. M. has been partially supported by the INFM project GEPAIGG01. T. M. acknowledges the Japan Scholarship Foundation. Numerical simulations have been performed at IDRIS (projects 011226 and 011411), at CINECA (INFM Parallel Computing Initiative), and at SX5 of the Yukawa Institute.

[1] A. S. Monin and A. M. Yaglom, Statistical Fluid Mechanics, I, 58 (1971)

[2] E. D. Siggia, Ann. Rev. Fluid Mech. 26, 137 (1994)

[3] G. Falkovich, K. Gawȩdzki, and M. Vergassola, Rev. Mod. Phys. 73, (2001) to appear

[4] A. Celani, A. Mazzino, and M. Vergassola, Phys. Fluids 13, 2133 (2001)

[5] D. Bernard, K. Gawędzki, and A. Kupiainen, J. Stat. Phys. 90, 519 (1998)

[6] A. Celani and M. Vergassola, Phys. Rev. Lett. 86, 424 (2001)

[7] I. Arad, L. Biferale, A. Celani, I. Procaccia, and M. Vergassola, Phys. Rev. Lett. 87, 164502 (2001)

[8] K. Gawȩdzki and A. Kupiainen, Phys. Rev. Lett. 75, 3834 (1995)

[9] M. Chertkov, G. Falkovich, I. Kolokolov, and V. Lebedev, Phys. Rev. E 52, 4924 (1995)

[10] B. I. Shraiman and E. D. Siggia, C. R. Acad. Sci. Paris, série II 321, 279 (1995)

[11] J. Paret and P. Tabeling, Phys. Fluids 10, 3126 (1998)

[12] G. Boffetta, A. Celani, and M. Vergassola, Phys. Rev. E 61, R29 (2000) 